University of Montana

ScholarWorks at University of Montana

$5-2009$

\title{
Ecological Facilitation May Drive Major Evolutionary Transitions
}

Zaal Kikvidze

Ragan M. Callaway

University of Montana - Missoula, Ray.Callaway@mso.umt.edu

Follow this and additional works at: https://scholarworks.umt.edu/biosci_pubs

Part of the Biology Commons

Let us know how access to this document benefits you.

\section{Recommended Citation}

Kikvidze, Zaal and Callaway, Ragan M., "Ecological Facilitation May Drive Major Evolutionary Transitions" (2009). Biological Sciences Faculty Publications. 93.

https://scholarworks.umt.edu/biosci_pubs/93

This Article is brought to you for free and open access by the Biological Sciences at ScholarWorks at University of Montana. It has been accepted for inclusion in Biological Sciences Faculty Publications by an authorized administrator of ScholarWorks at University of Montana. For more information, please contact

scholarworks@mso.umt.edu. 


\title{
Ecological Facilitation May Drive Major Evolutionary Transitions
}

\author{
ZAAL KIKVIDZE AND RAGAN M. CALLAWAY
}

\begin{abstract}
There is a growing consensus among ecologists that ecological facilitation comprises a historically overlooked but crucial suite of biotic interactions. Awareness of such positive interactions has recently led to substantial modifications in ecological theory. In this article we suggest how facilitation may be included in evolutionary theory. Natural selection based on competition provides a conceptually complete paradigm for speciation, but not for major evolutionary transitions - the emergence of new and more complex biological structures such as cells, organisms, and eusocial populations. We find that the successful theories developed to solve these specific problematic transitions show a consistent pattern: they focus on positive interactions. We argue that facilitation between individuals at different levels of biological organization can act as a cohesive force that generates a new level of organization with higher complexity and thus allows for major evolutionary transitions at all levels of biological hierarchy.
\end{abstract}

Keywords: positive biotic interactions, biological hierarchy, biological complexity, speciation, evolutionary theory

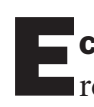
cology and evolutionary biology are two closely related disciplines, yet their theoretical directions do not always overlap. Therefore, an effort to synthesize the theoretical directions developing within these disciplines has the potential to provide crucial insight into biology's challenging issues. Because biotic interactions are the foundation for many important ecological and evolutionary concepts, including speciation, extinction, niche theory, and geographical distributions, they provide important interdisciplinary common ground. While ecologists studied the mechanisms of biotic interactions, evolutionists explored their genetic consequences. Here we suggest that recent ecological advances on understanding how species interact have important implications for evolutionary theory.

Ecological studies of biotic interactions have a long history, yet until recently, theories on coexistence, diversity-ecosystem function, meta-population dynamics, the niche, and the fundamental nature of communities have focused on negative interactions such as competition and predation, whereas positive interactions were considered to be interesting but idiosyncratic (Callaway 1997, Stachowicz 2001). Only since the 1990s has compelling evidence accrued for facilitation - that is, species interactions that are mutually beneficial—as a ubiquitous and important component of the suite of biotic interactions that determine fundamental ecological theory (Callaway 1998, 2007, Bruno et al. 2003, Brooker et al. 2008). Current models that include direct and indirect species interactions in ecological communities predict that positive interactions are as probable and as important as negative interactions (Callaway 2007).

Evolutionary theory has also explicitly based the origins of new lineages on negative biotic interactions, since the elimination of less fit individuals is very often the negative effect of one organism on another. But as in ecology, this focus has been criticized for the neglect of positive interactions as important drivers of evolution (Kutschera and Niklas 2004). The core of this criticism has been that novel biological structures cannot emerge unless functional links and cooperation, essentially positive interactions, occur among the components of a system. For example, the theory of natural selection on phenotypes resulting exclusively from mutations does not satisfactorily explain the rapid and important evolutionary transitions necessary to produce eukaryotic cells (Ryan 2002). Thus, most biologists now accept the endosymbiotic hypothesis, which explicitly bases the origins of eukaryotic cells on positive interactions (Margulis et al. 2000, Kooijman et al. 2003).

In fact, ecology's relatively recent shift in focus regarding biotic interactions in ecology has a parallel in evolutionary biology. In their book The Major Transitions in Evolution, John Maynard Smith and Eörs Szathmáry (1997) demonstrate how the problems that have proved difficult for mainstream 
evolutionary theory have been tackled by invoking new hypotheses that shift the focus from negative to positive interactions (also see Queller 1997). Maynard Smith and Szathmáry analyzed the strengths and weaknesses of evolutionary theory and argued that when evolution is presented as a series of major evolutionary transitions from less complex to more complex biological forms (eukaryotic cells are more complex than prokaryotic cells, animals and plants are more complex than protists, and so on), the theory of natural selection needs substantial modifications to predict or explain the emergence of new and more complex biological structures.

This problem becomes more evident if we superimpose evolutionary theory onto another general biological concept - the hierarchy of biological organization (figure 1, left panel). The hierarchical presentation of biological organization is heuristically useful because it portrays the complexity of all biological forms precisely and succinctly (McShea 2001).The general hierarchical pattern is "nestedness": higher levels of biological organization include lower levels (organisms include cells, cells include organelles, and so on). The major transitions refer precisely to these passages from one level of biological organization to another (figure 1, yellow arrows). A competition-based view of natural selection successfully explains emergence of biological diversity within levels, especially at the levels of cells and organisms, but a competition-based approach fails to satisfactorily explain the major transitions from lower to upper levels-and this pattern of nestedness, which portrays some of the most fundamental evolutionary changes in life.

The problem, as Maynard Smith and Szathmáry (1997) explained, is that natural selection based on negative interactions predicts competition between entities at the lower level (replicating molecules, free-living prokaryotes, single cells, individual organisms), which disrupts their ability to integrate into higher levels (chromosomes, eukaryotic cells, multicellular organisms, social structures). These important gaps left by the theory of natural selection have been filled in a rather haphazard manner by other biological theories, which developed independently to describe the emergence of each level of biological organization. One example is the endosymbiotic theory mentioned above (Margulis et al. 2000, Kooijman et al. 2003).

Two generalizations inferred from a large body of research on ecological facilitation apply directly to major evolutionary transitions. First, facilitative interactions become ecologically (and hence evolutionarily) meaningful in stressful environments where protection from environmental impacts is a principal general mechanism of facilitation (Callaway 2007, Brooker et al. 2008). Consequently, under certain conditions, the evolutionary process may be affected more strongly by facilitative interactions, and major evolutionary transitions may have been initiated in environments in which facilitation was important. The second generalization is that facilitating organisms aggregate closely with each other (Callaway 2007, Brooker et al. 2008); hence, major evolutionary transitions triggered by positive effects are clearly compatible with the nested hierarchy of life. Here we assess the potential of positive interactions to provide conceptual generality for major evolutionary transitions by discussing the importance of facilitation in the context of specific theories.

\section{Overview of specific theories on major evolutionary transitions}

The sequence of major evolutionary transitions, the most successful specific theories explaining these transitions, and the ecological interactions hypothesized to drive them are summarized in figure 1. Below we comment briefly on each of these transitions, from subcellular structures to socially organized populations. As this overview includes a broad range of biological hypotheses, we have presented them in a basic format that emphasizes fundamental underlying biotic interactions and, for brevity's sake, leaves out other important details.

Precellular evolution to simple cells. Precellular evolution includes at least two major evolutionary transitions: (1) from biomolecules to supermolecular aggregations such as chromosomes and ribosomes, and (2) from supermolecular aggregations to prokaryotic cells. No fossils were left by precellular transitions, and research relies mainly on the physics and chemistry of biomolecules, reconstructions of metabolic pathways for different evolutionary lineages, and theoretical modeling. The many hypotheses these studies produced are reviewed and discussed elsewhere (e.g., Knoll 2004, Martin and Russell 2007), but to date the leading theory is the "RNAworld" (Alberts et al. 2002). This hypothesis is based on the ability of RNA molecules (ribozymes) not only to self-replicate but also to catalyze necessary chemical reactions. Although a complete chemical reconstruction of self-replicating ribozymes is still to be achieved, the current hypothesis is consistent with the theory of hypercycles (Eigen and Schuster 1979). Hypercycles are new structures that emerge (nucleate) from interacting biomolecules such as nucleic acid chains, proteinoids, and lipids (Martin and Russell 2007). According to the stochastic corrector model (Szathmáry and Maynard Smith 1999), in which self-replicators represent sequences composed of a small number of building blocks (e.g., nucleotides), under certain conditions (new sequences enter the system only through copying other sequences that are already present, but incorrect inclusion of nucleotides is allowed, and raw materials for replications are always sufficiently available), these molecular complexes can form "quasispecies" (Eigen et al. 1989) that can participate in natural selection and may evolve gradually into cellular structures.

A brief synopsis of this theory for precellular evolution is that different biomolecules compete for resources (simpler chemical compounds that allow them to self-reproduce), but may also participate in positive interactions through the exchange of the products of chemical reactions specific to different biomolecules (Martin and Russell 2007). Under certain conditions as assumed by the stochastic corrector model, the selection jumps to an upper or group level (Szathmáry and 
Maynard Smith 1999). Importantly, after this transition, the usefulness of mutations is evaluated not from the point of view of a given individual, but from the point of view of the entire group. Consequently, a group becomes a new quasispecies but with a more complex structure that integrates the previous lower levels. As new quasi-species multiply, they compete strongly with each other and diverge into different lineages. In other words, competition between groups drives selection at the group level, whereas within groups, individuals facilitate each other; the transition is based on a certain, hierarchically structured interplay between negative and positive interactions. Such cycles could repeat more than one time and in more than one system. For example, through facilitative interactions, quasi-species could emerge from ribozymes, proteinoids, and lipids to develop into ribosomes, and in parallel, DNA chains could interact positively with other proteinoids and evolve into chromosomes. Consequently, through facilitative interactions, chromosomes and ribosomes could nucleate new quasi-species that evolve into prokaryotic cells. Prokaryotic cells represent compartments surrounded by well-developed outer membrane systems that protect genes and all the metabolic machinery; these cells are the first level of biological organization that left fossils (Cavalier-Smith 2006, Martin and Russel 2007).

From simple to complex cells. Many theories have competed to describe the transition from prokaryotic to eukaryotic cells, but the indisputable winner over the last three decades is the endosymbiotic theory mentioned above (Margulis et al. 2000, Kooijman et al. 2003). This theory is based on fossils, cell structure, metabolic pathways, genetic composition, and mathematical modeling (Margulis et al. 2000, Watson and Pollack 2003). The most accepted modern version is the serial endosymbiotic theory. This theory states that the evolution of eukaryotes from prokaryotes involved series of symbiotic unions of several previously independent ancestors, in which some independent organisms became organelles such as mitochondria and chloroplasts-and perhaps even nuclei, although the experimental evidence for this is not strong (Kooijman et al. 2003). The serial endosymbiotic theory explicitly bases these transitions on positive interactions that lead to a switch from natural selection acting on individual prokaryote cells to selection acting on cell unions (Watson and Pollack 2003). Also at this major transition we see a hierarchically structured interplay of positive and negative interactions: the cell unions survive and spread because cells engaging in endosymbiosis outcompete those that do not.
Interestingly, early versions of the endosymbiotic theory assumed that the evolution of complex cells started from parasitism (e.g., Cavalier-Smith 2006). However, parasitism as a starting point for symbiosis raises two issues that are difficult to explain. First, this presumes the existence of advanced, already complex, large cells that might host the hypothetical parasitic precursors of the organelles, and there is no evidence that such cells existed. Second, how do parasites become symbiotic? The necessity of an additional mechanistic process is not parsimonious. Even if parasitism has contributed to the origins of complex cells, these interactions between simple cells still may be classified as facilitative because facilitation does not exclude parasitism. There are many examples of benefactors experiencing competition and other negative effects from beneficiaries (Callaway 1994, 2007, Kikvidze et al. 2001).

From colonial to differentiated multicellular organisms. The classic theory of natural selection has been highly successful for explaining the evolution of different taxa of multicellular organisms. This level of biological organization has been intensively researched, producing elaborate phylogenies based on compelling evidence from fossils, embryology, physiology, biochemistry, and genetics. In general, these phylogenies divide multicellular organisms into three kingdoms: plants, animals, and fungi, and indicate a common ancestor from which these types of organisms have been originated: green algae for plants and spongelike organisms for animals and fungi (for reviews, see Graham et al. 2000 for plants, Dewel 2000 for animals, James et al. 2006 for fungi). These theories coincide in the idea that all of these lineages evolved from a colonial organism - an aggregation of identical, nondiffer- 
entiated cells. This is the stage at which the theory of natural selection could be revised to emphasize positive interactions. It is difficult to explain the origins of colonial organisms because ecological competition for resources should drive spatial dispersion among cells, not aggregation (Maynard Smith and Szathmáry 1997), or lead to the death of large proportions of the populations (Agusti et al. 1987). Nevertheless, colonies evolved and thrive today (Müller 2003).

The benefits of colonial lifestyles for cells are often emphasized (Solari et al. 2006), and these benefits are generally linked to the size of the colony - a larger size enables colonies to explore new niches, escape predation, and resist abiotic disturbance. The importance of positive interactions ("cooperation") among the cells of a colony, which start to differentiate and specialize in distinct functions, is also emphasized (Kirk 2005). These positive interactions within the colony can create selective pressures, leading to further evolution of cell differentiation (Michod 2007). Yet these ultimate benefits cannot provide proximate mechanisms by which the evolutionary transition from unicellular to colonial organisms occurs (Maynard Smith and Szathmáry 1997). Although agglomeration of cells to avoid predation could be considered a proximate common mechanism that drives selection for intercellular cooperation (Sachs 2008), this problem has not been fully addressed.

Herron and Michod (2007) conducted a thorough investigation on the origins of multicellularity in colonial algae. They derived a nearly complete phylogenetic reconstruction of the origins of multicellularity for volvocine algae that complements the hypothetical 12-step transitional chain elaborated earlier (Kirk 2005). Two striking features of this reconstruction are that (1) positive interactions and cooperation among cells at early steps were crucial for starting the evolution of multicellularity, and (2) when positive interactions weakened, colonies were disrupted and evolution of multicellularity reversed. Overall, the evolution of multicellularity has initiated and reversed more than once (Herron and Michod 2007), thus emphasizing the importance of positive interactions for the evolutionary transition from the unicellular to the multicellular form of life.

From individuals to superorganisms. Our arguments so far have explored the evolutionary transitions from molecules to multicellular organisms. Unfolding the potential of facilitation for evolutionary theory would not be complete without including social organization of populations, which also can be considered a product of the next major evolutionary transition (Maynard Smith and Szathmáry 1997). The most prominent products of this transition are the highly organized populations of social insects, such as bees and ants, often called "superorganisms" (Maynard Smith and Szathmáry 1997, Reeve and Hölldobler 2007). Superorganisms resemble multicellular differentiated organisms in that the specialized castes of individuals within superorganisms show division of functions similar to different cell types and tissues within the organisms (Maynard Smith and Szathmáry 1997, Jones and
Oldroyd 2007). This division of labor is genetically determined so that the colonies, and not their individual members, are units of evolution (Wilson 1975). For clarity we break down the evolution of superorganisms into two consecutive stages: first, the aggregation of individuals into groups, and second, the evolution of social (altruistic) behavior in superorganisms. Both stages pose specific difficulties for the theory of natural selection.

Natural selection predicts that individuals in a population will compete for resources, and hence losers must disperse away from winners in search of new space with resources (Maynard Smith and Szathmáry 1997). Many species conform to this prediction, but many other species form groups that gain social benefit from the group (e.g., flocks or herds). This inconsistency is addressed by a specific hypothesis of resource dispersion, which tries to explain aggregation in a group by the heterogeneity of the environment (Johnson et al. 2002). However, empirical evidence provides poor support for this hypothesis (Revilla 2003). We suggest that facilitation has good potential to resolve this problem: facilitative interactions that increase in intensity in stressful environments may explain the transition from solitary to group organization of populations, as discussed in the previous section for the transition from unicellular to colonial organisms.

Second, the organization of populations into groups and superorganisms requires the social (altruistic) behavior of individuals. Such behavior is evidently a product of evolution (Komdeur 2006), yet the theory of natural selection does not include altruism (Maynard Smith and Szathmáry 1997). This conflict has been resolved by kin selection, a modification of evolutionary theory (Hamilton 1964a, 1964b, Maynard Smith 1964). Kin selection predicts that when a group is genetically homogeneous, helping other individuals increases the probability of reproducing genes that beneficiary and benefactor individuals share. Another popular theory is that of group selection (Borello 2005, Reeve and Hölldobler 2007), which suggests that a transition of selection from individuals to groups is possible when there is a higher payoff for an individual to invest in helping individuals of the group than to invest in within-group competition. The approaches of kin and group selection are not exclusive and potentially can be combined (Boomsma and Franks 2006, Wilson and Wilson 2007), and both theories shift in focus from competitive to facilitative interactions.

As a final note, genetically determined division of labor can be found among organisms of different species: obligatory mutualisms present a clear example of such an above-organism level of biological organization. Obligatory mutualisms are often observed among very different biological forms (plantbacteria, plant-animal, animal-bacteria, fungi-algae, etc.; for reviews, see Simms and Taylor 2002, Bronstein et al. 2006, Kiers and van der Heijden 2006, Thrall et al. 2007), and evolutionary theory explains their origins by coevolution based on positive interactions. For example, the symbiotic interactions that produce lichens can actually accelerate the evolution of individual partner organisms (Lutzoni and Pagel 1997). 


\section{Facilitation in major evolutionary transitions}

Our analysis has followed the lead of earlier critics of theory for major evolutionary transitions. These critics suggested revisions in evolutionary theory to emphasize the importance of cooperation (positive interactions) for these transitions (Maynard Smith and Szathmáry 1997, Queller 1997). We have added analysis of the competing theories for major evolutionary transitions, and argued that seemingly idiosyncratic theories that were used to fill gaps in the theory of natural selection find cohesion and generalization in facilitation.

A detailed review of these theories is not possible in a short article, but even a brief overview indicates that the most successful theories-hypercycles, endosymbiosis, multicellularity, kin selection, and group selection-explicitly invoke positive biotic interactions (figure 1). The ecological equivalent of these interactions is facilitation, which is simple, can emerge spontaneously in environments under certain conditions, and may serve as a starting point for the evolution of the above-mentioned advanced types of positive interactions. A broader synthesis of specific biological theories for different aspects of natural selection may benefit from a more explicit focus on facilitation. One principal contribution from the ecological literature on facilitation is to provide a new way of perceiving the role of the environment in evolution. Environment not only filters out less fit individuals, but can create shifts from competitive interactions to facilitation. Facilitation and competition operate simultaneously (Callaway et al. 1991, Callaway 2007), but under stress, facilitation can increase the probability of survival of aggregated self-replicating entities more than competition can reduce their fitness. In this way, environmental conditions can alter the balance of facilitation and competition: within aggregated groups, the balance is shifted to facilitation, but between these groups competition prevails. This arrangement may provide the mechanism that triggers gradual transitions to higher levels of biological organization: although competitive interactions eliminate less fit groups, fitness can ultimately depend on the efficiency of facilitation within groups. Thus, ecological facilitation might advance cooperation and the division of function within aggregated groups of self-replicating entities. Hence, an explicit integration of ecological facilitation might contribute to the explanatory and predictive power of evolutionary theory, and, most importantly, to the evolution of large-scale increases in biological complexity.

Interestingly, the role of biotic interactions in the evolution of biological hierarchy may find a parallel pattern in organization of ecological systems. As it has been proposed, the hierarchy of interactions can structure many communities (Bruno and Bertness 2001, Baumeister and Callaway 2006, Altieri et al. 2007). For example, the formation of a community often starts with a "foundation species" that functions to engineer the habitat, which in turn facilitates an assemblage of other species, some of which may act as benefactors for other species. In turn, other types of biotic interactions, such as competition and predation, may further shape community organization within the habitat provided by the foundational species. In short, some ecological successions can be viewed as a cascade of positive and negative structure-driving interactions that interchange sequentially to create an intricate network of niches in an ecosystem (Altieri et al. 2007).

\section{Conclusion}

Overall, we find a striking parallel between ecology and evolution when considering the different roles of facilitative and competitive interactions in ecological processes and evolutionary transitions. This parallel involves common complex mechanisms that underpin biology and highlight the importance of synthetic theory. Perhaps at this stage positive effects deserve special attention, because the inclusion of facilitative interactions in important biological models can provide a powerful unifying generalization for explaining and predicting conceptually difficult transitional aspects of evolutionary theory, and may solve other major conceptual problems in which facilitative processes have not yet been considered.

\section{Acknowledgments}

The authors are grateful to Robin Brooker and Christopher Lortie for valuable discussion of these ideas, and to the Mellon Foundation for supporting our meetings.

\section{References cited}

Agusti S, Duarte CM, Kalff J. 1987. Algal cell-size and the maximum density and biomass of phytoplankton. Limnology and Oceanography 32: 983-986.

Alberts B, Johnson A, Lewis J, Raff M, Roberts K, Walter P. 2002. Molecular Biology of the Cell. 4th ed. Garland Science.

Altieri AH, Silliman BR, Bertness MD. 2007. Hierarchical organization via a facilitation cascade in intertidal cordgrass bed communities. American Naturalist 169: 195-206.

Baumeister D, Callaway RM. 2006. Facilitative effects of Pinus flexilis during succession: A hierarchy of mechanisms benefits other plant species. Ecology 87: 1816-1830.

Boomsma JJ, Franks NR. 2006. Social insects: From selfish genes to self organisation and beyond. Trends in Ecology and Evolution 21: 303-308.

Borello ME. 2005. The rise, fall and resurrection of group selection. Endeavor 29: 43-47.

Bronstein JL, Alarcon R, Geber M. 2006. The evolution of plant-insect mutualisms. New Phytologist 172: 412-428.

Brooker RW, et al. 2008. Facilitation in plant communities: The past, the present and the future. Journal of Ecology 96: 18-34.

Bruno JF, Bertness MD. 2001. Habitat modification and facilitation in benthic marine communities. Pages 201-218 in Bertness MD, Gaines SD, Hay ME, eds. Marine Community Ecology. Sinauer.

Bruno JF, Stachowicz JJ, Bertness MD. 2003. Inclusion of facilitation into ecological theory. Trends in Ecology and Evolution 18: 119-125.

Callaway RM. 1994. Facilitative and interfering effects of Arthrocnemum subterminale on winter annuals in California salt marsh. Ecology 75 681-686.

- 1997. Positive interactions in plant communities and the individualistic-continuum concept. Oecologia 112: 143-149.

- 1998. Competition and facilitation on elevation gradients in subalpine forests of the northern Rocky Mountains, USA. Oikos 82: 561-573.

- 2007. Positive interactions and interdependence in plant communities. Springer. 
Callaway RM, Nadkarni NM, Mahall BE. 1991. Facilitating and interfering effects of Quercus douglasii in central California. Ecology 72: 1484-1499.

Cavalier-Smith T. 2006. Cell evolution and Earth history: Stasis and revolution. Philosophical Transactions of the Royal Society B 361: 969-1006.

Dewel RA. 2000. Colonial origin for Eumetazoa: Major morphological transitions and the origin of bilaterian complexity. Journal of Morphology 243: 35-74.

Eigen M, Schuster P. 1979. The hypercycle: A principle of natural selforganization. Springer.

Eigen M, McCaskill J, Schuster P. 1989. The molecular quasi-species. Advances in Chemical Physics 75: 149-263.

Graham LE, Cook ME, Busse JS. 2000. The origin of plants: Body plan changes contributing to a major evolutionary radiation. Proceedings of the National Academy of Sciences 97: 4535-4540.

Hamilton WD. 1964a. The genetical evolution of social behaviour, I. Journal of Theoretical Biology 7: 1-16.

- 1964b. The genetical evolution of social behaviour, II. Journal of Theoretical Biology 7: 17-52.

Herron MD, Michod RE. 2007. Evolution of complexity in the volvocine algae: Transitions in individuality through Darwin's eye. Evolution 62: 436-451.

James TY, et al. 2006. Reconstructing the early evolution of fungi using a six-gene phylogeny. Nature 443: 818-822.

Johnson DDP, Kays R, Blackwell PG, MacDonald DW. 2002. Does the resource dispersion hypothesis explain group living? Trends in Ecology and Evolution 17: 563-570.

Jones JC, Oldroyd BP. 2007. Nest thermoregulation in social insects. Advances in Insect Physiology 33: 153-191.

Kiers ET, van der Heijden MGA. 2006. Mutualistic stability in the arbuscular mycorrhizal symbiosis: Exploring hypotheses of evolutionary cooperation. Ecology 87: 1627-1636.

Kikvidze Z, Khetsuriani L, Kikodze D, Callaway RM. 2001. Facilitation and interference in subalpine meadows of the Caucasus. Journal of Vegetation Science 12: 833-838.

Kirk DL. 2005. A twelve-step program for evolving multicellularity and a division of labor. Bioessays 27: 299-310.

Knoll AH. 2004. Life on a Young Planet: The First Three Billion Years of Evolution on Earth. Princeton University Press.

Komdeur J. 2006. Variation in individual investment strategies among social animals. Ethology 112: 729-747.

Kooijman SALM, Auger P, Poggiale JC, Kooi BW. 2003. Quantitative steps in symbiogenesis and the evolution of homeostasis. Biological Reviews 78: 435-463.

Kutschera U, Niklas KJ. 2004. The modern theory of biological evolution: An expanded synthesis. Naturwissenschaften 91: 255-276.

Lutzoni F, Pagel M. 1997. Accelerated evolution as a consequence of transitions to mutualism. Proceedings of the National Academy of Sciences 94: $11422-11427$.

Margulis L, Dolan MF, Guerrero R. 2000. The chimeric eukaryote: Origin of the nucleus from the karyomastigont in amitochondriate protists. Proceedings of the National Academy of Sciences 97: 6954-6959.

Martin W, Russell MJ. 2007. On the origin of biochemistry at an alkaline hydrothermal vent. Philosophical Transactions of the Royal Society B 362: 1887-1925.
Maynard Smith J. 1964. Group selection and kin selection. Nature 201: 1145-1147.

Maynard Smith J, Szathmáry E. 1997. The Major Transitions in Evolution. Oxford University Press.

McShea DW. 2001. The minor transitions in hierarchical evolution and the question of a directional bias. Journal of Evolutionary Biology 14: 502-518.

Michod RE. 2007. Evolution of individuality during the transition from unicellular to multicellular life. Proceedings of the National Academy of Sciences 104: 8613-8618.

Müller WEG. 2003. The origin of metazoan complexity: Porifera as integrated animals. Integrative and Comparative Biology 43: 3-10.

Queller IDC. 1997. Cooperators since life began, review of J. Maynard Smith and E. Szathmáry, The Major Transitions in Evolution. Quarterly Review of Biology 72: 184-188.

Reeve HK, Hölldobler B. 2007. The emergence of a superorganism through intergroup competition. Proceedings of the National Academy of Sciences 104: 9736-9740.

Revilla E. 2003. Moving beyond the resource dispersion hypothesis. Trends in Ecology and Evolution 18: 380.

Ryan F. 2002. Darwin's Blind Spot: Evolution Beyond Natural Selection. Houghton Mifflin.

Sachs JL. 2008. Resolving the first steps to multicellularity. Trends in Ecology and Evolution 23: 245-248.

Simms EL, Taylor DL. 2002. Partner choice in nitrogen-fixation mutualisms of legumes and rhizobia. Integrative and Comparative Biology 42: 369-380.

Solari CA, Ganguly S, Kessler JO, Michod RE, Goldstein RE. 2006. Multicellularity and the functional interdependence of motility and molecular transport. Proceedings of the National Academy of Sciences 103: 1353-1358.

Stachowicz JJ. 2001. Mutualism, facilitation, and the structure of ecological communities. BioScience 51: 235-246.

Szathmáry E, Maynard Smith J. 1999. The Origins of Life. Oxford University Press.

Thrall PH, Hochberg ME, Burdon JJ, Bever JD. 2007 Coevolution of symbiotic mutualists and parasites in a community context. Trends in Ecology and Evolution 22: 120-126.

Watson RA, Pollack JB. 2003. A computational model of symbiotic composition in evolutionary transitions. Biosystems 69: 187-209.

Wilson DS, Wilson EO. 2007. Rethinking the theoretical foundation of sociobiology. Quarterly Review of Biology 82: 327-348.

Wilson EO. 1975. Sociobiology: The New Synthesis. Harvard University Press.

Zaal Kikvidze (e-mail: zaal@k.u-tokyo.ac.jp) is with the University of Tokyo in Kashiwa, Japan, and also with the Department of Life Sciences at Ilia State University in Tbilisi, Georgia. Ragan M. Callaway(e-mail: ray.callaway@mso. umt.edu) is with the Division of Biological Sciences at the University of Montana in Missoula. 